\title{
Applied Research on the Supervision Standardization of 750kV Transformer Substation Engineering--Based on the Special Geology and Environment in China's Northwest Area
}

\author{
Jun $\mathrm{Qi}^{1, \mathrm{a}}$, Yu Shan ${ }^{2, \mathrm{~b}}$, Lu Zheng ${ }^{3, \mathrm{c}}$ \\ ${ }^{1}$ Xinjiang Electric Power Engineering Supervision Co., Ltd, 830000 \\ ${ }^{2}$ National network of Xinjiang Institute of supervision company \\ ${ }^{3}$ Xinjiang Electric Power Engineering Supervision Co., Ltd, 830000 \\ 68872388@qq.com ${ }^{\mathrm{a}}$, 2694678468@qq.com ${ }^{\mathrm{b}}, 516185216 @ q q . c o m^{c}$
}

Key words: Supervision, Standardization, Innovation

\begin{abstract}
V}$ substation project in China's northwest area is located in the edge of the desert, and in the site, there are many depressions, besides, the topsoil salinization phenomenon is relatively common and the crystallographic white salt efflorescence is visible. The depth of groundwater can reach to $2.1 \sim 4.0 \mathrm{~m}$, water quality is very poor and the water is salt water, which belongs to brackish water and it is cannot be used as production water and drinking water. In the site, the groundwater and site soil have strong corrosivity to the concrete structure. The overall objective of the project is to achieve the China Power Quality Engineering Award and National Quality Project Award. Based on the special geological environment and high demand for the engineering in the site and after constant exploration and practice,the special supervision standardization working mode forms directing at the engineering items, quality and safety management and we has made significant effect in the process of engineering management.
\end{abstract}

\section{Introduction}

After years of conclusion on theory and practice, the developed capitalist countries have made relatively mature engineering construction supervision standards. They apply all aspects of knowledge and methods to the standard and guide and standardize the supervision personnel and their activities with the standard, besides, evaluate the supervision work so that to improve the business level of supervision teams and the overall quality of the activities.

For this objective research, based on the supervision specification and supervision standardization handbook issued by SGC, according to the actual site situation of $750 \mathrm{kV}$ substation project and after continuous exploration and research, it achieves the application of supervision management standardization system in this engineering, besides, through the application of standardized process, it achieves the purpose of final inspection quality control results.

\section{Main Application}

This subject, based on the supervision management work of northwest area $750 \mathrm{kV}$ substation engineering, takes innovation practice and specific instruction from three aspects of quality, safety and project management, besides, directing at various extented innovation content, in the similar engineerings and project management of the same industry, it can both effectively apply the standardization innovation system, further more, under the condition of effective resources, it can bring great benefits for project management and construction investment. 


\section{Innovation spot}

For engineering site types of groundwater pore type diving, there is local perched at the same time. The site is dug to a depth more than $4 \mathrm{~m}$ flashing phenomenon, has led to the completion of the excavation foundation diffuse water, seriously damaged base completed tank cleaning work. After the field study, the light well point dewatering before excavation is adopted to improve the construction quality and schedule. As a quality management unit, the supervision party through the site topography, construction side well point precipitation scheme, design drawings, the following data were summarized in the table format and through the relevant data monitoring and control, and ultimately determine whether the groundwater is ruled out and the excavation of the official time. The main data of the monitoring include: pump head, the number of light points, the depth of the well point, the pump switch time, the daily output of water, the total amount of water, the total length of the water.

Corrosion inspection of concrete foundation with strong corrosive geology. Because of the particularity of the regional geological environment in the project, the quality of the soil and water are alkaline corrosion, Groundwater has strong corrosive to the concrete structure and reinforced concrete structure, The ground soil has strong corrosive to the concrete structure, the steel bar in the reinforced concrete structure has the medium corrosion, and the steel structure has strong corrosion resistance. According to the design requirements, the concrete base cushion is used in the asphalt lake to increase the corrosion resistance. The periphery of the foundation with gravel subgrade replacement of raw soil, and on the basis of the surface corrosion of asphalt layer, improve the overall quality of anticorrosion. In view of the above process quality construction, the supervision policy of asphalt concrete management has developed a statistical method of relevant parameters, form the source to the construction of the inspection table. The specific parameters include asphalt concrete mix ratio, raw material producing area, raw material factory test report, raw material factory temperature, raw material construction temperature, thickness of cushion thickness measurement, the whole process to ensure the quality of bedding. On the basis of the surface coating, take the production of specimens, try to form a surface besmear to brush paint, paint the number of statistical data, through theoretical data and practice combined, and through the spiral micrometer determination way eventually determine the specific coating process.

Installation quality control of large scale equipment. For better control of the whole process of equipment installation quality, to strengthen the supervision of equipment for the installation of each step of the familiar degree and the key processes of the key to control. According to the manufacturer's instructions, equipment provided by truck transport scheme, construction units reporting scheme, equipment installation drawings, industry rules, develop equipment installation inspection card", it covers the basis of self inspection, equipment installation in front of the truck, and electric transmission check before a total of more than 10, through the full range of process control, to ensure effective lifting equipment installation quality.

Green environmental protection control in large sandy area. Because the project is located at the edge of the desert, for many years the prevalence of monsoon, in order to better do a good job in the construction process of environmental protection and water conservation work, the supervisor according to the project characteristics and progress of the situation, the development of the "three kinds of table" application: Civil construction phase of the green construction inspection table; electrical equipment installation phase of green construction inspection table; green inspection table. In the three class examination table, on human factors and environmental factors (temperature, wind direction, wind, dust, noise, dust) productivity factors analysis, formulate unified compilation, reason analysis and Countermeasures table. The final summary of the table data can provide a reliable reference for the meteorological department and the land department to analyze the local environmental water and soil factors.

Management of testing instruments based on fine and lean. For scientific, rational, standardized use and management of equipment, while ensuring that all kinds of testing equipment 
can really be used for engineering, services in the project, and to ensure the quality of the on-site inspection and testing in place, the instrument name, specification design, site location, measurement value, the measured value, the use of time, the scene photos used for unified assembly, to establish "use account instruments". This is the "fusion equipment ledger ledger" + "on-site inspection record", which brings great convenience for site management.

Engineering digital photo directory. In order to strengthen the management of engineering image files and improve the integrity and the scientific nature of the digital photo management of the State Grid Corporation. According to the national network company digital photo management approach to the quality of the 322 text, security 25, to create the northwest regional $750 \mathrm{kV}$ substation project digital photo shoot". And according to the characteristics of the project entity, the key parts, new technology and new applications, new materials, and other important hidden processes to enhance the shot, improve quality, safety process management.

Application of project management enterprise lean platform and remote video platform. Based on the enterprise internal management engineering department of the projects required, the establishment of the platform, which include platform upload dynamic update, the project supervision memorabilia, digital photo management, engineering supervision on-site inspection document writing and timely upload, the company management personnel at home in case of a comprehensive understanding of dynamic engineering. The use of this lean system, can form a mutual learning environment between the various projects of the company, and ultimately promote the competition situation among the project department, better promote the efficient operation of the platform and practical application. With the help of remote video platform, realize the entity dynamic monitoring project, can help the staff in addition to enter the construction site outside the time effective monitoring of the construction site, the introduction of video capture function, making the scene of the violation of the quality and safety of the work becomes nowhere to hide, can not only improve the supervision efficiency, and enhance the quality of the control field.

Household registration in personnel management. In view of the Xinjiang autonomous region to fully carry out the stability of the stability of the situation, the scene of all personnel to adopt a comprehensive policy of household registration, the establishment of dynamic information database including the name of the person, the provinces domicile, nationality, political affiliation, identity card number, health (medical institutions), residence permit, cultural degree, work experience, skill level, and no criminal record and personal credit evaluation information. Indicating the personnel approach and departure time, and make sure, cards, books, contracts, certificates of conformity for unity, to a certain extent prevent subcontracters shelter evil people and countenance evil practices to fulfill social responsibility. Based on the application of this system, the use of fingerprint identification, face recognition system, unified personnel into and out of management, to ensure that the personnel information on the site is accurate and effective.

Grid management and the internal mutual inspection system. In view of enhancing the engineering quality and supervision personnel professional theory, practical knowledge of the dual consideration, on-site supervision and control to carry out the grid, mutual inspection model development. For the 750 thousand volt substation area is large, according to the function of the regional geographical location as a basis for the distinction, and professional geographic regions within the division, and finally to the professional foundation, carry out regional management, does not regularly carry out between different regions of the same professional personnel exchange check system, in order to ultimately enhance the professional knowledge and engineering entity quality personnel. Such as: Northwest Region 750 thousand volt substation distribution between 750 thousand volt and 220 thousand volt and 66 thousand volt transformer and outdoor switchgear, civil engineering supervision division, A B, according to the format of network management mode, A is responsible for the construction work area of 750 thousand volts, 220 thousand volts, B is responsible for the main transformer and 66 thousand volt soil construction work, the implementation of mutual inspection system in the normal management of the normal area under the jurisdiction of the case. Namely: A and B do not regularly carry out personnel work on, eventually to the internal appraisal, experience on ways to achieve double harvest quality, improve 
the quality of personnel. Through the network division of labor, a clear responsibility of the subject; through the implementation of mutual inspection system to enhance the overall quality.

Defect library information platform application. In the network times, the characteristic of information is high efficiency, convenience and sharing. As for the power industry quality engineering award, a national high quality project award of the project as a whole, the quality supervision departments should reflect the characteristics of the project. The engineering project supervision department as the main body, to create the project site, the formation of several large plate project schedule, engineering, planning, standard process of milepost display etc. While most of the characteristics of the plate is "build defect library", defects in the base content improved, the project supervision department to collect the company over the years, Xinjiang Xinjiang with large-scale engineering, similar engineering professional engineering intermediate acceptance defects, the prosecution operation defects, defects are collected to form defects of excellence, library, and all Xinjiang Regional Publicity on Web site, in the construction process management for supervision, construction and design units comprehensive reference, in order to reduce up to eliminate the same defect happen again. And increase the message board in the defect library column, enhance the ability and experience to deal with difficult defects by means of communication and interaction.

\section{Application Prospect}

In the standardization system, as a way of checking and correcting the deviation and deviation in the process of management, it is the most direct. Such as: the process of green construction inspection and local conditions of light well point precipitation project, the green construction of the content of the inspection and rectification can play a direct role in environmental protection, light well point precipitation under the premise of local conditions, can save a lot of manpower and material resources, and effectively save expenses, etc. To enhance the detection of key points of the key points, invisible to reduce human, material, material, such as blind waste.

\section{Conclusion}

The research of supervision standardization can be effectively implemented in the application of the same kind of engineering. For the same industry engineering and the same nature of the project, can be directly applied to. As a kind of standardization research of engineering, it is a kind of practice and promotion to the management culture of enterprise and project department.

\section{Reference}

[1] Kunrun Qian, Maotian Wang, Jieming Zhao—Concise supervisor's manual[M] China Architecture \& Building Press,2000

[2] Guojun $\mathrm{Ke}$-Building engineering construction supervision staff post series[M] China Architecture \& Building Press,2005

[3] Tianzhen Gong, Zeping Zhang___ntroduction to construction engineering supervision[M] Peking University Press,2006

[4] Haimin Wei, Junyao Zheng_Construction engineering supervision practice[M] China Planning Press,2006

Jun Qi(1971-)male, Han nationality, Jining Shandong people, Xinjiang Electric Power Engineering Supervision Co., Ltd. Director of power network supervision department Tel: 18999991975 e-mail:68872388@qq.com

Mailing address: No. 123, Jianshe Road, Urumqi, Xinjiang

Yu Shan(1974-)male, Han nationality, Shanxian County Shandong people, National network of 
Xinjiang Institute of supervision company Director general e-mail: 2694678468@qq.com

Mailing address: No. 123, Jianshe Road, Urumqi, Xinjiang

Lu Zheng(1986-)male, Han nationality, Fuping Shanxi people, Xinjiang Electric Power Engineering Supervision Co., Ltd. Director representative Tel: 15999070741 e-mail: 516185216@qq.com Mailing address: No. 123, Jianshe Road, Urumqi, Xinjiang

邮寄地址：新疆乌鲁木齐市建设路 123 号 15999070741 